\title{
An Auction-Based Recommender System for Over-The-Top Platform
}

\author{
Hameed AlQaheri ${ }^{1, *}$, Anjan Bandyopadhay ${ }^{2}$, Debolina Nath ${ }^{2}$, Shreyanta Kar $^{2}$ and \\ Arunangshu Banerjee 2
}

\author{
${ }^{1}$ Kuwait University, Kuwait \\ ${ }^{2}$ Amity University Kolkata, Kolkata, 700135, India \\ *Corresponding Author: Hameed AlQaheri. Email: hameed.alqaheri@ku.edu.kw \\ Received: 08 July 2021; Accepted: 12 August 2021
}

\begin{abstract}
In this era of digital domination, it is fit to say that individuals are more inclined towards viewership on online platforms due to the wide variety and the scope of individual preferences it provides. In the past few years, there has been a massive growth in the popularity of Over-The-Top platforms, with an increasing number of consumers adapting to them. The Covid-19 pandemic has also caused the proliferation of these services as people are restricted to their homes. Consumers are often in a dilemma about which subscription plan to choose, and this is where a recommendation system makes their task easy. The Subscription recommendation system allows potential users to pick the most suitable and convenient plan for their daily consumption from diverse OTT platforms. The economic equilibrium behind allocating these resources follows a unique voting and bidding system propped by us in this paper. The system is dependent on two types of individuals, type 1 seeking the recommendation plan, and type 2 suggesting it. In our study, the system collaborates with the latter who participate in voting and invest/bid in the available options, keeping in mind the user preferences. This architecture runs on an interface where the candidates can login to participate at their convenience. As a result, selective participants are awarded monetary gains considering the rules of the suggested mechanism, and the most voted subscription plan gets recommended to the user.
\end{abstract}

Keywords: Recommendation systems; over-the-top platforms; subscription allocation; auction theory

\section{Introduction}

Over the past decade, the entertainment industry of the nation has undergone several changes. This phenomenon is due to the increase in the easy availability and access to internet services and devices such as smartphones, desktops, laptops, tablets, smart-TVs, etc., which can interconnect the Internet easily. Media services are not just limited to television and theatres anymore. With the increase in demand for online resources, OTT (Over-The-Top) platforms are the recent favorite of all. The advent of the unprecedented pandemic situation caused by the sudden outbreak of COVID-19 has brought in much alteration in the way audience consume media [1]. The sudden

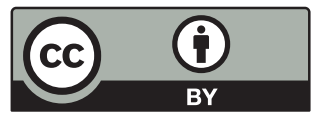

This work is licensed under a Creative Commons Attribution 4.0 International License, which permits unrestricted use, distribution, and reproduction in any medium, provided the original work is properly cited. 
imposition of the much-needed lockdown throughout the nation and the emergence of the social distancing norms have taken the audience away from cinema halls, theatres, and auditoriums and restricted them to their houses. However, the demand for entertainment and media has not been curbed, thereby increasing the popularity of the OTT platforms in the nation [2]. There has been a massive boom in the number of subscribers on the OTT platforms in recent times. They have evolved as highly dominating entertainment platforms for consumers in the country, as shown in Fig. 1. OTTs serve the platter based on the choice and preference of the consumer in a pocketfriendly budget. Hence, it has evolved as a massive entertainment platform for the masses.

Statistics and various other research reports point to the growing market and consumer appetite for the content of choice available on OTT platforms [3]. OTTs offer a never before consumer advantage-choice of content, ease of access, choice of device/mediums (handphone, laptop, tablet, or TV screen). Gone are the days when family members fought for screen time of choice on the family's singular home device, i.e., TV. Urban cities, in particular, find it quite common for almost all members of a family to get access to individual smartphones/laptops. As every consumer has his/her taste in content viewership, OTTs allow them to do so, on their device without adjusting or compromising for someone else.

Number of paid subscribers(in million) on different OTT platforms in India

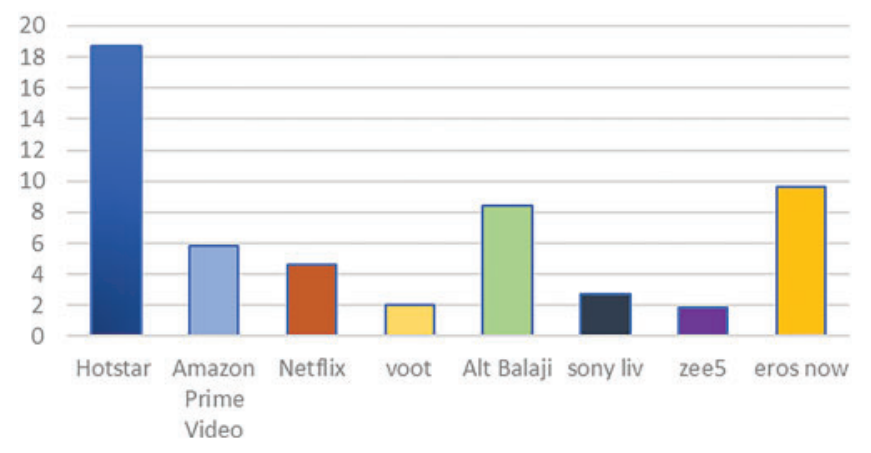

Figure 1: No. of paid subscribers in different OTT platforms in India

Now, the consumers can opt for the subscription plan of their own choice based on their budget and the content they want to view. In this scenario, every consumer is looking for the best possible subscription plan for their preferences as per the conditions set by them. Recommendation systems have made this process easy by giving the users the best suggestions based on their preferences. Everyone is looking for maximum benefits on a minimum budget, and it will indeed be an additional gain for them if they get an opportunity to get their subscription plan at a lower price in exchange for their participation in a voting competition to choose the most recommended subscription plan on an OTT platform. Most architectures use a preference-based algorithm and concepts like collaborative and content-based filtering to generate the recommendation. A unique concept is implemented in our recommendation system that uses the concepts of voting and bidding systems to generate the result and the winning amount, respectively.

Our paper proposes a unique mechanism in which users can gain cashback or buy their monthly subscriptions at a discounted price simply by participating in a voting contest conducted in a certain time frame. The format contains two types of users: 
(1) Consumers-individuals who require the recommendation.

(2) Participants-individuals who will vote for a particular service based on the user's requirements and participate in the game.

The participants are to vote for the OTT subscription plan, which they think is the best according to the conditions set by the consumer. Suppose the OTT subscription that they voted most for emerges as the winner of the contest. In that case, the participants who voted for it will be winning some cash back or discount on their subscription plan thereby benefitting him/her and cutting down this expenditure. The most voted plan will be recommended to the consumer. This process browses through all the available/popular OTT platforms to ensure the most accurate plan is suggested that suit the interests of the user. The system model explained in detail in the further sections of the paper can be correlated to a lottery system. The buyer of the lottery ticket invests some amount of money purchasing the ticket and if he/she wins, then gets back much more than the amount invested. The winning amount that he/she gets is given from the total amount collected from the participants (i.e., amounts invested by each of the buyers who purchased the ticket). We design a similar model to deal with the voting contests conducted for the OTT platforms.

The calculation of the reward will be based on a unique bidding system modeled by us. In our system, we are implementing the concept of this unique auction mechanism to determine the prize money to be received by the winning participants. Here, not only the highest bidder gets rewarded, but the second-highest bidder also receives a reward amount, taking into consideration that only those bidders who voted for the winning subscription plan. A similar concept has been deployed in the problem statement to calculate the winning amount. The detailed mechanism of the bidding system and collection of bid values has been discussed in Section 3 of the paper. Thus, to engage the participants in voting, we deploy this method. The participants investing their money will have a chance to win an amount back, encouraging them to involve themselves in this system. At the same time, the consumer in need of the suggestion will also get the desired result.

The rest of the paper is structured as follows: some previous works in this field are discussed in Section 2, comprising Literature Review. Section 3 formulated the system model and system architecture followed by the Proposed Mechanism in Section 4 where we present the proposed solution for the subscription recommendation system in detail. In Section 5, we assign a statistical analysis based on our model. In Section 6, we visualize the simulation results that we have obtained using our system. In Section 7, we have mentioned the tool used for the implementation, Lastly, we present the limitations and advantages of our work and highlight some future applications using cloud computing in Section 8 of the paper, comprising Conclusion and Future Work.

\section{Literature Review}

The advancement of technology has promoted the use of internet-based delivery among audiences for entertainment purposes. In turn, OTT (Over-the-top) media service platforms have emerged to be one of the leading providers of viewership today. They are a growing space of competition, network, regulation, and development, among other advantages [4]. With the Covid19 pandemic at large, India is anticipated to become the second biggest OTT market, closely followed by the USA [5-7]. Thus, statistical analysis of user preferences over consumer data has helped visualize acceptance between different platforms and content alike [5,6]. With growing demand, global hotshots like Netflix, Hulu, and Amazon Prime have also learned to share a room 
with Indian streaming services like Hotstar and Voot. The existence of these media services is to bring great changes to the economy, now that cinema halls are mostly restricted from use. The familiarity, demand, and availability surrounding the collective watching experience are only expected to grow more with time.

Streaming services are relatively cost-effective with the right choice of platform and package. In April 2019, a study revealed that more than 50\% of users experience "subscription fatigue" from signing up on too many subscription services. It may also impact the position of smaller services against established platforms like Netflix, Hotstar, etc. Thus, survival analysis techniques came into play, using data from ratings, reviews, and recommendation systems. This hypothesis led to the discovery of purchase probability maximization to help extend the subscription period for their customers. It observes the history of long-term purchase patterns to increase user satisfaction and benefit online businesses [8]. As these platforms operate in a digital space, there is an abundance of information at hand. Thus, the Internet has been dependent on recommendation systems of different algorithms to build that trust and effective facilitation among potential customers [9].

Machine learning using recommendation systems is a popular and fascinating area of research in the IT world. Today, some of the widely known applications of this approach can be found in suggesting movies, music, news, etc., to potential consumers [10]. Search engines, social media accounts, and websites also follow recommendation algorithms by monitoring online activities for the best user experience. Thus, the need for research surrounding this topic has been inactive pursue in the last decade.

The theory of collaborative filtering suggests that two users A and B are likely to have the same preferences if they harbor familiar opinions and tastes over a commodity [11]. Similarly, content-based filtering also revolves around user preferences while prioritizing collected data over human collaborations. In short, we can say that collaborative filtering is driven by consciously made choices, while content-based filtering administers data over time and inspection. Related sub-categories like memory-based and model-based systems can also be found in the collaborative filtering approach [12]. By combining these collaborative and content-based filtering with others, administrators have long overcome many glitches in their application in a hybrid approach. Compared with the hybrid architecture, multi-criteria recommender systems (MCRS) [11] explore preferential information from the users for more than one criteria or value. However, like the others [11,12], even the multi-criteria matrix is not immune to a cold start. Thus, it brings the other forms of work in this field into play.

Sparse and inefficient data at an early stage are but a few of those prevailing issues today. Hence, instead of the above methods, certain implementations believe in using a context-based filtering approach [13] for minimizing the required data and information to customize it according to the situation or user demands. The leverage that context-based mechanism has over contentbased and collaborative filtering is the consideration of time in their examination. With contextual factors like time and purpose in the play, static and dynamic methods can better predict the stability of user feedback [13].

Reinforcement learning theory follows a reward-based system for the recommended agent by exposing it to a real-life environment [13]. The economic setting in an auction theory also follows a similar reward-based architecture that allows the participants to make financial trades in a market-based approach. Unlike our former studies in this section, it encourages collecting the latest information from the users, in turn aiding the allocation of reliable services that keeps updating themselves with the trends. This method also resolves errors that arise from a cold start, 
sparsity, and inefficient data. Thus, the two-way transactions in a bidding environment are more likely to fetch rewards and clarity for the client and organizer's outcome.

Auction theory is a sub-part of game theory [14]. Some popularly known algorithms in this topic are the first and second-price auction theory algorithm shown in [15,16]. It is primarily targeted towards generating the most profit for the participants and system controller. While the first price auction theory is relatively simple and straightforward, making sure the highest bidder is at the end of the winning deal, the second price auction theory focuses on the second-highest bid for its formulation [16]. Another concept that is also included in this mechanism is called the reverse auction theory [17]. In this approach, the roles of the buyer and seller get altered, giving the algorithm its name. This system pitches one buyer against various potential sellers willing to sell their goods or products. The sellers here change their prices according to the buyer's demand. Next, the sellers compete with each other to offer the best possible price that is effective and inexpensive, such that the buyer gets convinced to buy the product. Another difference of approach from the above auction theory is that the sellers generally tend to decrease the price so that it is feasible for the buyer to purchase the project. In contrast, in the case of a traditional auction, the bid values generally tend to increase.

Some other popularly known auctions are Open-ascending bid Auctions or English auctions [18] and Open-descending bid auctions or Dutch Auctions [19]. In the case of an English auction, the seller sets an opening price for a particular product, and bidders start to bid their values, which is greater than the opening amount. The person with the highest bid wins. In the case of a Dutch auction, generally, it is started with a high price by the seller, and then this price decreases gradually until any bidder agrees with the price. Once any bidder accepts the price, the auction ends. Thus, in general, the auction architecture helps achieve financial incentives from financial investments.

\section{The Conceptual Auction-Based Recommender System for Over-The-Top}

\subsection{System Model and Problem Formulation}

This section presents the working model of the unique Recommendation system we have formulated for OTT platforms, with simultaneous Voting and Bidding system, as proposed in Section 1. The problem will be dealt with in a certain time frame, thereby taking a dynamic approach.

This system will involve two categories of users:

- First, a consumer $C$ who is giving the preferences and asking for a recommendation

- Second, a set of participants $X=\left\{x_{1}, x_{2}, x_{3} \ldots x_{n}\right\}$ who are bidding and voting based on the requirements laid down by the consumer.

The consumer sets the conditions such as price range, quality, device connection, content preference, etc., as per his or her choice and then asks for the recommendation for the most suitable subscription plan based on popular choice. Let $\mathrm{P}$ be the set of subscription plans available for the consumer, satisfying all the conditions set by the consumer, such that $P=\left\{P_{1}, P_{2}, P_{3} \ldots P_{m}\right\}$, where $\mathrm{m}$ is the number of subscription plans available. The consumer now uses the recommendation system to choose the most suitable subscription plan from the set $\mathrm{P}$.

Once the consumer seeks the recommendation, the system controller starts voting and bidding within the recommendation system. According to the problem statement, each game will be restricted to a fixed time frame $\mathrm{T}$ (taking $\mathrm{T}=15 \mathrm{~min}$ ), after which another new game will be 
initiated. In this time frame, the participants can vote for any one subscription plan, which they can choose according to their opinion from the set of available subscriptions $\mathrm{P}$. The participants urge to participate in the voting contest with the expectation of winning cashback. To participate in the voting, each participant has to invest a certain bid value in the auction from a set of four bid values $b$, such that $b=\left\{b_{1}, b_{2}, b_{3}, b_{4}\right\}$ and $b_{1}>b_{2}>b_{3}>b_{4}$. The system in every time-frame will randomly generate these bid values. The auction will be conducted on a new set of bid values generated by the system every time in every time frame. It makes it a real-time dynamic system capable of working perfectly with changing conditions. All the voters/bidders have to vote and pay their bid values within the given time frame $T$, exceeding which will result in disqualification.

After the completion of the voting and bidding in time-frame $T$, as set by the system controller, the system will calculate the total number of votes obtained by each subscription plan belonging to set $\mathrm{P}$ and the plan getting the maximum votes will emerge as the winner and that plan will get recommended to the consumer $\mathrm{C}$. Then the distribution of the prize money to the winning participants will be carried out by the system, for this calculation of the prize money and distribution among the winning participants.

To illustrate this system, let us consider the following example:

Let us say $P_{1}$ has received $n_{1}$ votes, $P_{2}$ has received $n_{2}$ votes, and $P_{3}$ has received $n_{3}$ votes where $\mathrm{n}_{3}>\mathrm{n}_{2}>\mathrm{n}_{1}$.

Thus, in this case, the winner is $\mathrm{P}_{3}$, and plan $\mathrm{P}_{3}$ gets recommended to consumer $\mathrm{C}$.

The highest number of votes is equivalent to $\mathrm{n}_{3}$, which corresponds to the number of winners. Now, out of these $n_{3}$ winners, all must not have invested the same bid value. In our approach, only the winners who have invested the highest and the second-highest bid value, i.e., b1 and b2, will be receiving their winning amounts.

The winning amount for each participant belonging to these two categories of winning participants will be calculated as follows:

- The participant who invested in $b_{1}: r_{1} * b_{1}$ (where $r_{1}$ lies in the range 1.25 to 1.50 )

- The participant who invested in $b_{2}: r_{2} * b_{2}$ (where $r_{2}$ lies in the range 1.10 to 1.25 )

Both $r_{1}$ and $r 2$ are randomly generated by the system for every time frame, restricting the values of $r_{1}$ and $r_{2}$ to their specific ranges.

However, the remaining winning participants, i.e., those who had invested b3 and b4 bid values, respectively, will get not be losing their invested amounts. Instead, they will get straight cashback of the amount they invested, thereby making no profit any loss. This system will encourage them to stay in this recommendation system for a longer time and urge them to participate further in the voting and bidding game.

Here we consider that $\mathrm{y}_{1}, \mathrm{y}_{2}, \mathrm{y}_{3}$, and $\mathrm{y}_{4}$ are the number of participants who paid the bid values $b_{1}, b_{2}, b_{3}$, and $b_{4}$ for the winning plan $P_{3}$.

The formulation of the equations for the total prize money, winning amount, and system's profit amount is below:

- Total collected amount $=\mathrm{T}$

- Amount won by winning highest bidders $=\mathrm{y}_{1} * \mathrm{r}_{1} * \mathrm{~b}_{1}$

- Amount won by winning second highest bidders $=\mathrm{y}_{2} * \mathrm{r}_{2} * \mathrm{~b}_{2}$ 
- Amount repaid $=\left(\mathrm{y}_{3} * \mathrm{~b}_{3}\right)+\left(\mathrm{y}_{4} * \mathrm{~b}_{4}\right)$

- Amount received by the system $=\mathrm{T}-\left\{\left(\mathrm{y}_{1} * \mathrm{r}_{1} * \mathrm{~b}_{1}\right)+\left(\mathrm{y}_{2} * \mathrm{r}_{2} * \mathrm{~b}_{2}\right)+\left(\mathrm{y}_{3} * \mathrm{~b}_{3}\right)+\left(\mathrm{y}_{4} * \mathrm{~b}_{4}\right)\right\}$

The total amount $\mathrm{T}$ that is collected by the system will be the summation of the bid values paid by each of the participants in the game. From this collected amount, the winning amount is distributed among the winning participants. After all the distributions, the amount remaining from the total collection is kept by the system controller as the system's profit. Thus, this system benefits winning participants and gives adequate profit to the controller or conductor of the system.

\subsection{The System Architecture}

Fig. 2, illustrates the working of the RAVA architecture in the form of a flowchart. It represents the sequence of events followed by the system model in one iteration or cycle. As we can see, here P1, P2, and P3 are the available subscription plans for which that algorithm has been established. The bidders, b1, b2, b3, and b4 are the estimated candidates involved in the auction process. This algorithm follows a novel auction system, which is a sub expression of game theory in computer science [14]. The mechanism assigns $n 1, n 2, n 3$, i.e., the votes obtained to each subscription plan, in turn deriving the winning plan chosen by the participants. Among these voters, the winners are then distinguished on the basis of their investment in the process. The highest and second highest bidders are then awarded their due incentives, while the other winners get their money returned back. An advantage of the latter is that interested people can access the mechanism with negligible losses before they can transcend to higher bidding amounts. It builds an understanding and familiarity with the model thereafter. In this scenario, revenue for the system controller is also generated alongside the participants. The algorithm makes sure that under every circumstance or iteration, the returns for the system controller remain unchanged by a marginal gap. The winning plan is henceforth allocated to the consumer, who requested for it. On repetition, the next cycle replaces the bidding values, b1, b2, b3, b4, with new values that are randomly chosen by the system within a range of rules. This system model runs on an interface, from where potential candidates can access the bidding contests as per their convenience. As the timeframe of the auction is already fixed, it also allows the algorithm to return results in a short span of time, instead of waiting on more bidders to join. Hence, we can say that our system architecture is both consumer and candidate friendly.

Fig. 3, illustrates the voting system used in this algorithm. This approach draws its idea from a concept of game theory [14], the Borda Voting system [20], which is also known as the System of Borda Count. In this voting system, each voter ranks each available subscription plan according to their choice. Each of the ranks is associated with a certain number of points/scores taken into account while counting the total scores obtained by a certain subscription plan. As described in the Fig. 3, the votes are being calculated by multiplying the number of voters and the points associated with each rank and then they are summed up to obtained the total number of votes. The total votes for each plan i.e., P1, P2 and P3 are being calculated in the same way. The plan that obtains the maximum points or scores emerges as the winner. The winning plan gets recommended to the consumer. 




Figure 2: Diagrammatic representation of our system

BORDA VOTING

\begin{tabular}{|l|l|l|l|}
\hline Voters & 76 & 44 & 25 \\
\hline $1^{\text {st }}$ choice: 5 pts & P1 & P3 & P2 \\
\hline $2^{\text {nd }}$ choice: 3 pts & P3 & P2 & P1 \\
\hline $3^{\text {rd }}$ choice: 1 pt & P2 & P1 & P3 \\
\hline
\end{tabular}

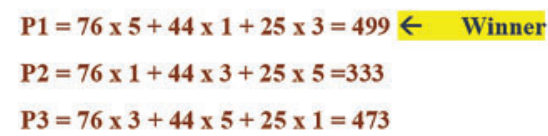

Figure 3: Representation of Borda voting system

\section{Proposed Mechanism}

The proposed mechanism for executing the System model discussed in Section 3 is based on a unique algorithm designed by us called Recommendation Algorithm on Voting and Auction (RAVA). On completion of this algorithm, the consumers will be recommended the Over-The-Top subscription plan, according to their provided preferences. The voters who had ranked the winning plan as their first choice will get rewarded according to the system discussed in the previous section. The pseudo-code of our working algorithm (RAVA) has been given below along with the detailed list of the variables used in the code: 







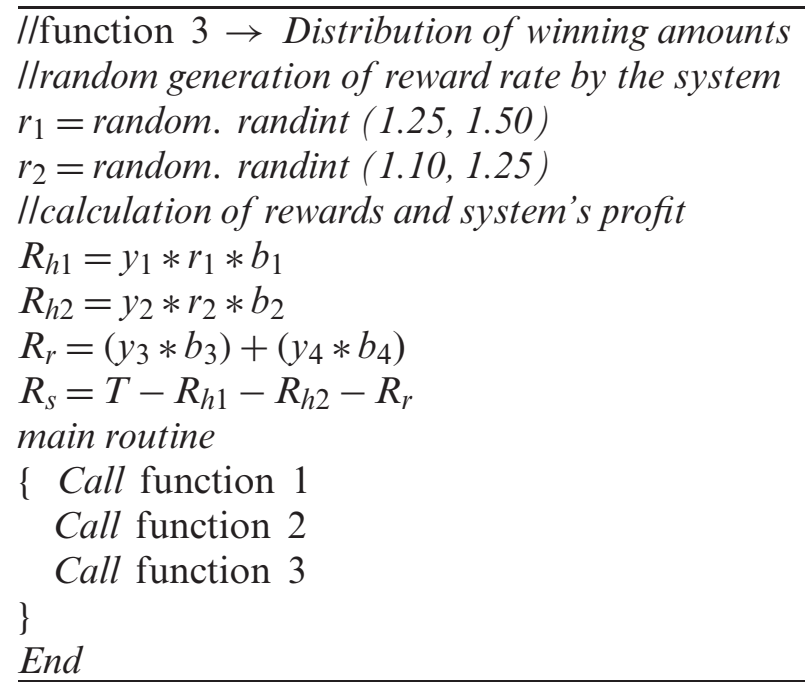

Time Complexity: The time Complexity of the given algorithm is $\mathrm{O}(\mathrm{n} * \mathrm{~m})$ since the loop runs for each value in the range 1 to $n$ where $n$ is the number of participants and within this loop, votes are received for $\mathrm{m}$ number of subscriptions plans available for each participant.

By measuring market metrics based on certain objectives, the performance of the system architecture or design can be evaluated efficiently. They fulfill criteria that are important for the success of the mechanism and prove the standards of the product in the market. In this problem statement, the recommendation system identifies the properties that a good auction environment should exhibit. Thus, with this theoretical and numeric data, we can say that our design is suitable to be introduced and put to use in the assigned platform. The following properties expand on the goals that our model completes to indicate its effectiveness in a real-world scenario:

Proposition 1. The RAVA mechanism is Pareto efficient.

Proof. Pareto efficiency can be defined as an economic circumstance where the condition of an individual cannot be made better or improved without making another individual's conditions worse. Following our proposal, it can be stated that to increase any participant's reward. We don't need to decrease anyone else's reward amount. There is no such situation in our proposed idea where any other participant's compensation is being compromised to multiply others prize amount. The rewards are generated as per the bid values invested by the participants with a calculated amount of profit for the highest bidder, second highest bidder, as well as the system controllers. Since this amount has a particular formula for calculation which is mentioned in the system formulation part. There are no such circumstances where there is the scope of alteration of these values. Thus, the reward amounts are generated as per the bid values and the votes received by the system. Hence, we can say our proposed system is Pareto efficient.

Proposition 2. The allocation by RAVA works towards producing Social welfare maximization.

Proof. The context of our paper pitches social welfare as a mechanism that will provide a way of distinguishing between Pareto efficient solutions to the given problem by determining which subscription plan is best for a recommendation from the point of view of the voters (which can be considered as a social viewpoint). In contrast to Pareto efficiency, social welfare provides a way to rank different subscription plan preferences over all the available subscription plans and to indicate the best-suited option for the subscription plan as a whole. It acts as a supplement to Pareto efficiency. 
Proposition 3. The RAVA architecture supports Individual rationality.

Proof. A mechanism is said to possess individual rationality when all its participants have an individually rational logic behind involving themselves in the bargain [21]. If a system provides an amount less than what was invested by the agents during the bid, the system is not rational. However, in our model, the least price gained by a winner is the same amount that he has bid during the auction. We can also say that while the highest winning bidder receives the maximum reward at the end of his bargain, the minimum reward generated by any winner is the same as what they have pitched. Thus, individual rationality has been taken into consideration in the evaluation of this model. This, in turn, encourages more people to participate and give the protocol a try as they have negligible losses to incur. If it weren't for this property, the system would be a victim to a cold start, and many valuable recommendations would be lost due to lack of participation.

Proposition 4. The RAVA system provides Clear Incentives to all the agents.

Proof. In this case, a mechanism should act so that all its agents can profit from its earnings globally. This property ensures the profit of the system controller with every bid, besides just safeguarding the earnings of the participants involved. The same feedback is thus expected, with a change in bid values or profit margin. The auction theory deployed in our approach makes sure that even with the fluctuation in incentives, the overall range between the highest winning bidder and system controller remains low. It helps maximize the revenue and provides each agent involved in the system their due prize.

Proposition 5. The proposed market in RAVA is Convergent.

Proof. The market is termed convergent when the prices associated with commodities tend to converge after a certain number of rounds of auction. In the context of our paper, the OTT market in which we are auctioning can be stated as convergent as the bid values allocated to the subscription plans tends to converge after certain rounds of voting and auction. This is very significant from the point of view of the bidders/participants. It allows them to analyze and learn how to bid rationally for a certain subscription plan so that they can extract maximum profits/reward amounts in exchange for their invested bid values. The property of convergence prevents the market behavior from becoming chaotic because it puts a sense of understanding into the bidders regarding how much to bid for a subscription plan in the recommendation system.

Proposition 6. The RAVA system model is stable in nature.

Proof. If a system behaves in a particular manner in which it is designed over time, it is stable. A protocol needs to stay under control irrespective of changing conditions. The output should always be right or proper with each input. Thus, the result shows that a stable system gives bounded output for bounded input. A stable system safeguards the best interests of all the individuals participating in the innovation. The performance evaluation in this design shows that even with multiple conditions in play, the profit will never turn out to be zero for any player. In detail, we can say that even with slight fluctuation in the profit margin or bidding values, the resultant gain for the participants, system controller, or customers is lost. Thus, the architecture pitched in this model is in full control of its input and output. 
Proposition 7. The RAVA mechanism promotes fair monetization.

Proof. A market should be fair to its participants. It should provide equal opportunity to provide opinions and earn rewards to the candidates [22]. The method used should also be unbiased in its efforts to provide the promised incentives. Therefore, keeping in mind these rules, we design a mechanism that serves the same purpose and promotes a fair auction environment. In this algorithm, incentives are directly proportional to the investments. Taking the best points from the first and second-price auction theory into consideration, our mechanism conducts an unbiased system, which returns the highest profit to the highest bidder while returning the same amount to the lowest. Thus, equal opportunity for all also gets established. If the system provides the same rewards for different auctioned prices, the profit earned would be unjust to the highest winning bidders, which is effectively eradicated by this mechanism, as proved above.

Proposition 8. The recommendation generated by RAVA is truthful to its users.

Proof. A mechanism is said to be truthful when it provides the same results to the users that it generates from the market research. The genuineness of the product is also dependent on how the reward is integrated into the system. Though money is a motivator to provide honest opinions to the customers, it can often be led by misplaced greed. Many bidders might curb their perspective to follow the majority and even indulge in fixing the auction environment. However, to avoid this mishap, our algorithm generates randomized values that refresh with a certain time frame. Hence, the resultant produced from this architecture, in the end, is honest and truthful to the clients.

The above points in theory and practice help a system achieve market equilibrium from an economic point of view. Though some of the points are purely seen from a numeric perspective, some of these in hypothesis make a system more authentic and market-ready for launch. The quality and standard of the model can also be evaluated from the feedback, leaving no question for the system's performance in a real platform or environment. Further, an analysis of the model and its working can be found in the later section of the paper for evaluation.

\section{Implementation and Simulation Results}

The above-mentioned algorithm has been executed on Windows 10 64-bit operating system with 8 GB RAM, and i5 processor. Python 3.9.1 has been used for the implementation of the given algorithm and the generation of the graphs using different sets of data. It took $0.938 \mathrm{~s}$ to generate the required output by the system after the voting and auction is completed in the timeframe of $15 \mathrm{~min}$ as discussed earlier.

The following are the simulation results that we have obtained from the successful execution of our proposed mechanism: -

The graph is shown in Fig. 4 shows the variations in the four bid values for the subscription plans that are randomly generated by the system in each time frame of auction. Here, the results can be seen for five different time frames in each of which the system assigns different bid values.

Fig. 5, shows the number of participants taking part in the voting and bidding in each of the five respective time-frames as mentioned. Along with the number of participants, the profits earned by the system, in every time frame, are also plotted. The plotted data reflect that the greater the number of participants involved in the voting system, the more profit is generated. 


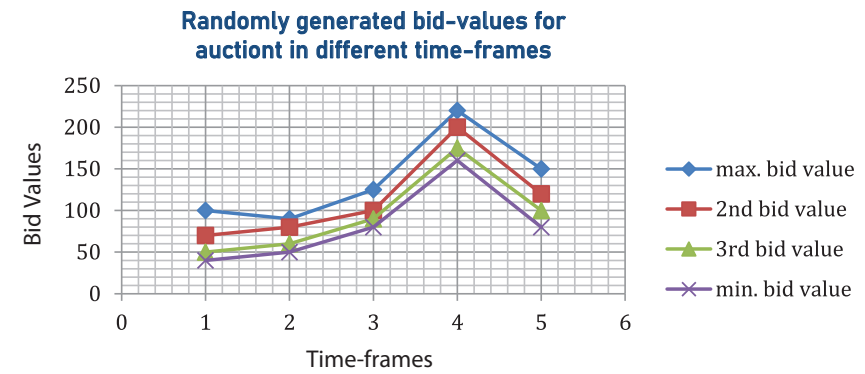

Figure 4: Variations in the four bid values over different time-frames

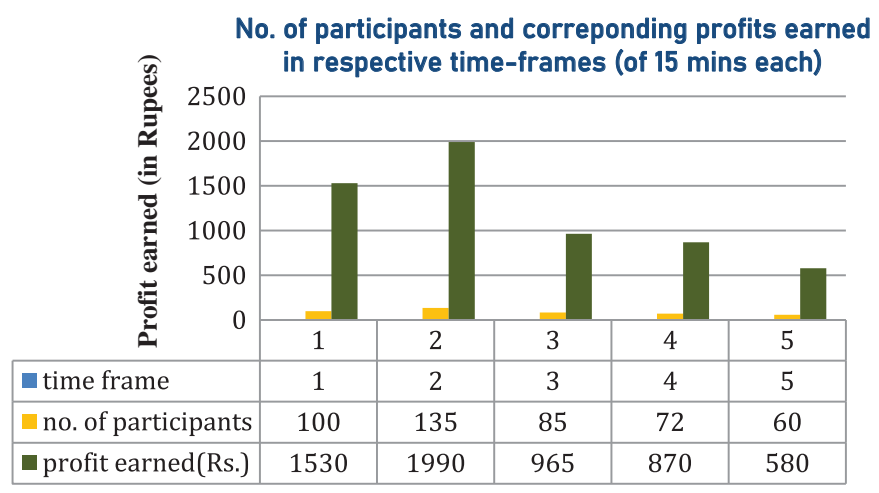

Figure 5: Number of participants taking part in the voting and bidding in each of the five respective time-frames

In Fig. 6, the plots have been made concentrating only on the auction occurring in TimeFrame 1 of duration $15 \mathrm{~min}$. In the graph, the total number of participants who took part in the voting is clearly shown, along with the number of participants who voted for the respective bid values. For this time frame, the bid values generated by the system were Rs. 100, Rs. 80, Rs. 60 and Rs. 50 respectively. As depicted, in a total of 100 participants, 60 voted for plan 1, and 40 voted for plan 2. Out of 60 who voted for subscription plan 1, 25 voted for the maximum bid value while the remaining 22,8 , and 5 participants invested in bid values 2,3 , and 4 , respectively. Also, out of 40 who voted for subscription plan 2, 20 voted for the maximum bid value while the remaining 10, 7, and 3 participants invested in bid values 2, 3, and 4, respectively. The winning subscription plan can be easily seen since the subscription plan with the maximum votes is the winner, i.e., subscription plan 1 in this case.

In Fig. 7, the plots have been made concentrating only on the auction occurring in TimeFrame 2 of duration $15 \mathrm{~min}$. For this time frame, the bid values generated by the system were Rs. 152, Rs. 112, Rs. 96 and Rs. 75 respectively. As depicted, in a total of 135 participants, 38 voted for plan 1, 52 voted for plan 2, and 45 voted for plan 3. Out of 38 who voted for subscription plan $1,17,22,8$, and 5 participants invested in bid values 1, 2, 3, and 4, respectively. Out of 52 who voted for subscription plan 2, 31 voted for the maximum bid value while the remaining 12,6 , and 3 participants invested in bid values 2, 3, and 4, respectively. Out of 45 who voted for subscription plan $3,25,22,8$, and 5 participants invested bid values $1,2,3$, and 4, respectively. 
The winning subscription plan can be easily seen since the subscription plan with the maximum votes is the winner, i.e., subscription plan 2 in this case.

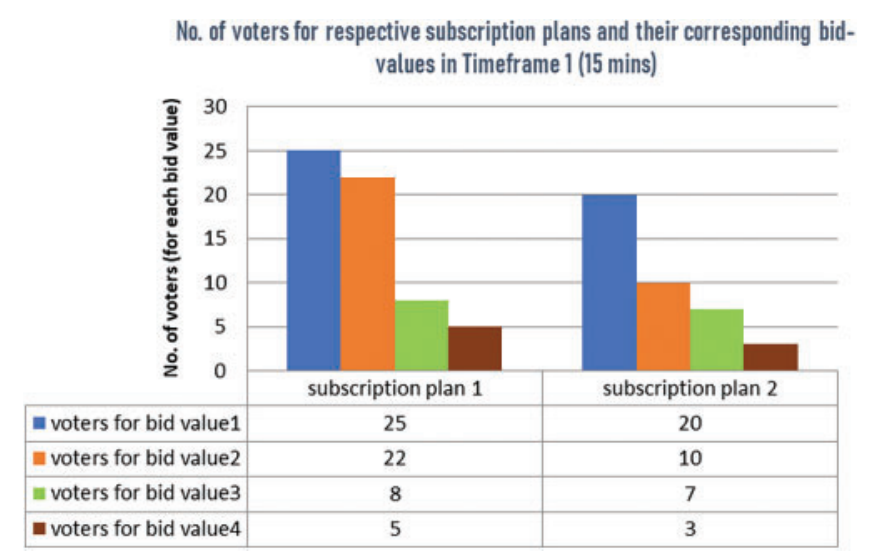

Figure 6: Number of voters for respective subscription plans in Time-frame 1



Figure 7: Number of voters for respective subscription plans in time-frame 2

In Fig. 8, the plots have been made concentrating only on the auction occurring in TimeFrame 3 of duration $15 \mathrm{~min}$. For this time frame, the bid values generated by the system were Rs. 85, Rs. 75, Rs. 65 and Rs. 55 respectively. As depicted, in a total of 85 participants, 33 voted for plan 1, and 52 voted for plan 2. Out of 33 who voted for subscription plan 1, 25 voted for the maximum bid value while the remaining 22, 8 , and 5 participants invested in bid values 2, 3, and 4, respectively. Also, out of 52 who voted for subscription plans, 2, 12, 28, 9, and 3 participants invested in bid values 1,2, 3, and 4, respectively. From here the winning subscription plan can be easily seen since the subscription plan which has the maximum votes is the winner, i.e., subscription plan 2 in this case.

In Fig. 9, similar plots have been made concentrating only on the action occurring in an extended Time-Frame of duration of $30 \mathrm{~min}$. This extended time frame is taken to involve 
more participants in the recommendation system to earn more profit. The effect of the extended timeframe of the auction can be clearly seen from the plotted graph as it indicates the increased number of participants as well as greater profits.

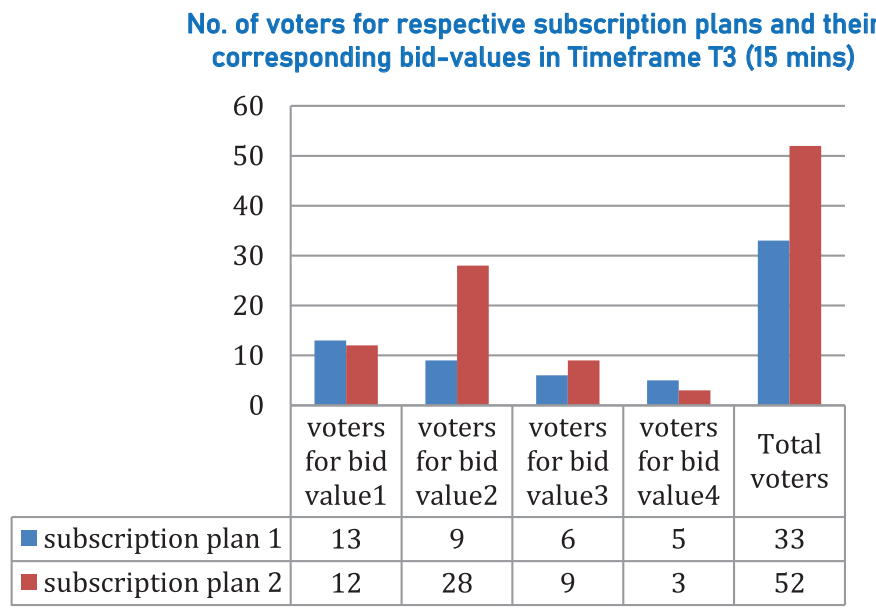

Figure 8: Number of voters for respective subscription plans in Time-frame 3

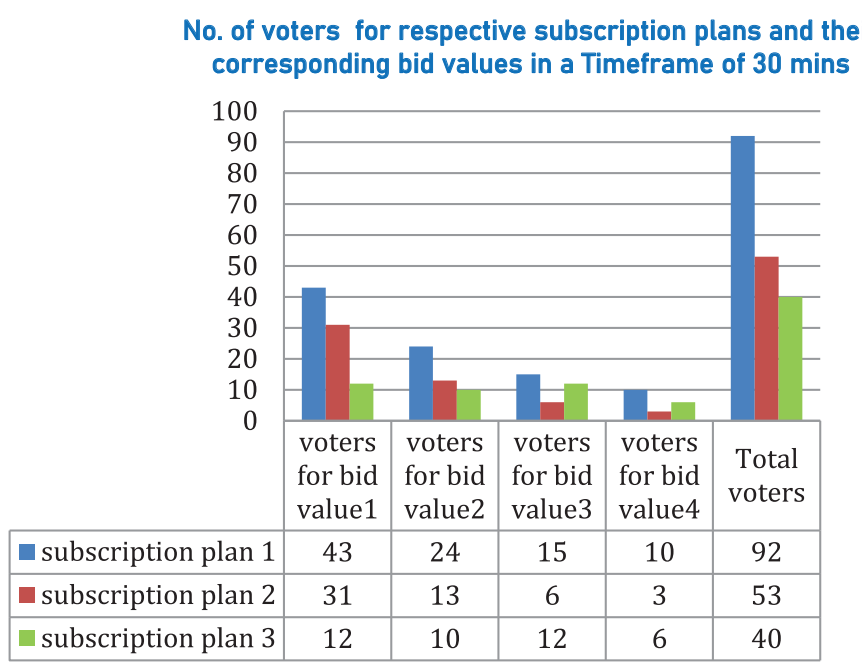

Figure 9: Number of voters for respective subscription plans in extended Time-frame

\section{Comparative Study}

Due to rapid digitalization in the recent years, there has been a global expansion in the consumption of Over-the-Top (OTT) platforms. In addition, the Covid-19 pandemic has also provided a considerable contribution in the number of users and hours spent that has led to the proliferation of these streaming services. 
South Korea is one of the leading nations in producing OTT content and it is seen that the South Korean audience also has high preference for OTT platforms. Some of the popular OTT platforms are Oksusu, pooq, Netflix, Wavve, Tving etc. Though the popularity of different OTT platforms varies from nation to nation, a common trend is seen in the rising number of subscriptions, OTT viewership and generated revenue. In this paper, we have compared the statistics of South Korean OTT services against Indian platforms over the years [23]. They cover the user subscriptions and revenue generation to understand and predict the consumption expectancy in the future.

The survey data presented here are collected from the internet source as available on the web. In Fig. 10, the graph shows the difference between the estimated subscribers, with India leading over Korea by a huge margin. Over the course of 2018-22, Korean OTT platforms can barely make it past 10 million users, while India shares a great raise in users around the same time. There is also a large difference analyzed in user subscriptions between 2018 and 2022, in India, by itself.

While the online revenue generation at South Korea had taken a great start due to the fact that South Korea had entered this market prior to India, India has considerably exceeded their profits with methods that worked well with the audience. Hence, though the monetary progress of South Korea is slow and steady, India is still leading over them in this area and has full potential to emerge as the most profitable nation in terms of OTT revenue and OTT market.

Fig. 10, shows the consistent rise of both countries, with visible marginal differences with each other and amongst themselves. The revenue generated in Fig. 11 according to this data is directly proportional to the number of subscribers we found in Fig. 10, as shown in the graphical representations. This distinction owns to the fact that India has more opportunity and popularity, surrounding digital originals. Many platforms often provide early streaming of live shows that are a hit among the audience. These techniques have proved to pave the way for online content superiority among viewers.

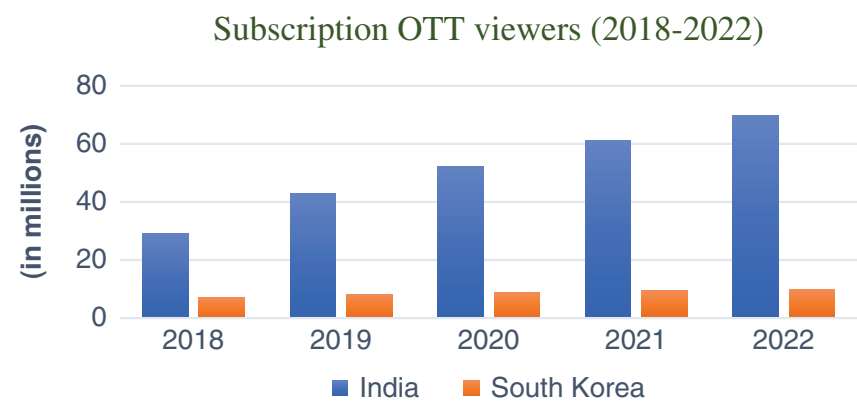

Figure 10: A comparison between the estimated number of subscribed viewers on OTT platforms in India and South Korea in 2018-2022

The Middle East/North Africa (MENA) region and in particular Saudi Arabia have observed some of the highest digital penetration and user consumption over the last five years and more. Hence, it makes the market trends suitable for the introduction of new services and SVoD expansion. After the declaration of Netflix joining the MENA markets in 2016, many operators like Google Play Movies, OSN, MBC, etc., all followed suit. Data collected in 2017 also suggests that people have shown more interest in opting for digital content over physical content by a margin 
of $1 \%$, post these changes. However, Saudi Arabia is yet to come up with its own platforms and digital originals that can impact the number of users for their content, locally or globally. On the other hand, India is dominated by several platforms like Hotstar, Voot, AltBalaji, JioCinema, etc., which are native to the country and its audience. With digital originals like Mirzapur, Sacred Games, etc., Indian content has successfully established its grounds in the global OTT space.

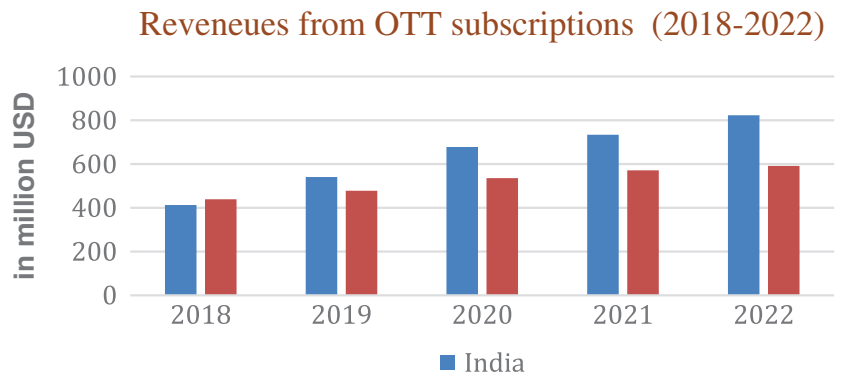

Figure 11: A comparison between the amount of revenue obtained from OTT platforms in India and South Korea in 2018-2022

\section{OTT Market Share in Saudi Arabia}

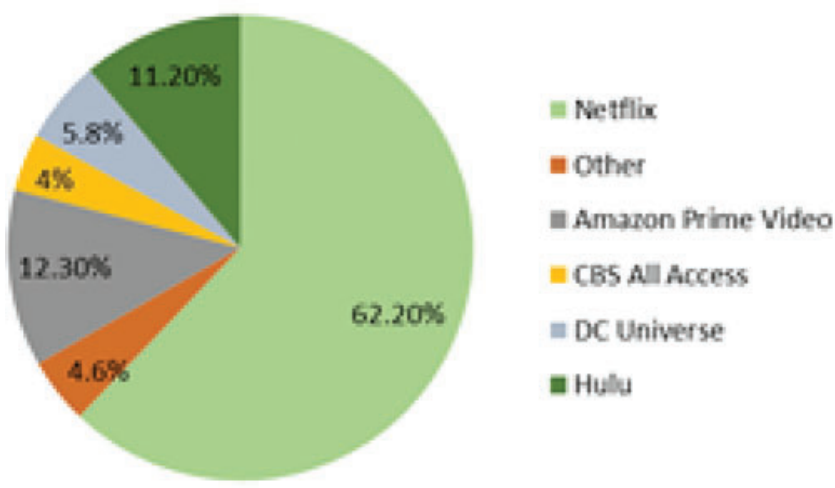

\section{Market Share of OTT Platforms in India}

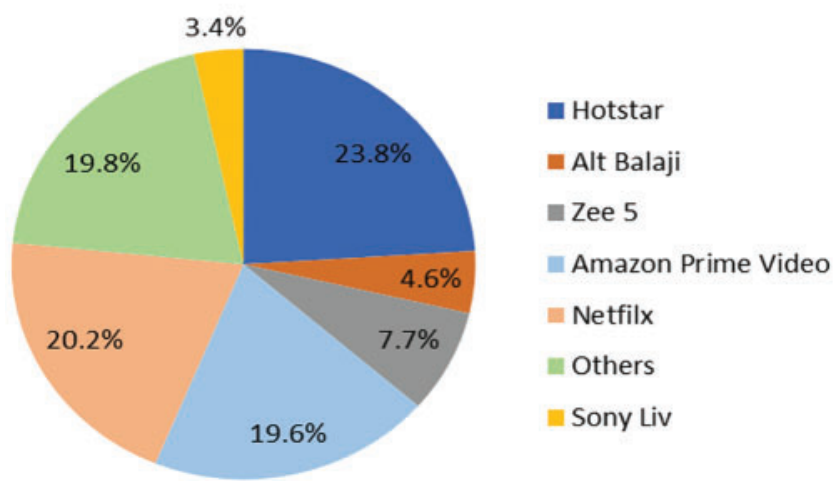

Figure 12: A comparison between the market share of OTT platforms in Saudi Arabia and India 
In our study, we have dealt with the consumption of premium services over free trials, which come with a definite subscription package for monthly or yearly use. The survey data presented here are collected from The Global Television Demand Report as available on the web. In Fig. 12 we can see that while the audience of Saudi Arabia is highly dependent on Netflix for its content at $62.20 \%$, India observes Hotstar leading over the former by a small margin of $3.6 \%$. Though the popularity of Netflix is unparalleled globally, the inclination of the Indian audience towards their native resources is a piece of good news for the overall revenue in the market share. Other noteworthy examples of use include Amazon Prime at $12.30 \%$ and Hulu at $11.20 \%$ in Saudi Arabia. Indian audience proves to be interested in a variety of platforms, with a $19.8 \%$ lead at category "Others", which is closely followed up by Amazon Prime at 19.6\%.

The graph in Fig. 13 represents the growth of subscribers in the industry, in correspondence to their population. Though there is a huge difference in inhabitants between India and Saudi Arabia, the statistics for the number of subscriptions between 2018-22, do not fare very well for the latter. The progress is expected to be consistent, but slow, which should have relatively sped up with the incoming of the Covid-19 pandemic. Some factors that might be affecting or delaying this development are a narrow range of original content, failure to produce native platforms, screen quality, and pricing plans.

On contrary, India shows immense potential in this sector, with a steadily increasing graph in the years to come. The estimated number of subscriptions alongside other factors is a direct indication of the revenue generated through the use of these platforms. Thus, it is only wise to say that India is most likely to profit at the pace it is moving.

Our recommendation system works towards balancing consumer spend with consumer experience, which makes it a beneficial tool for any platform provider or country to overcome their issues. This mechanism picks the right subscription plan for the users, in turn encouraging more users to associate with these platforms. Thus, it can act as leverage to the already increasing OTT market. Taking into view the increasing popularity and the rising trend for OTT subscriptions we can say that OTT platforms are the future of the entertainment industry worldwide. Thus, our proposed model stands true to benefit the users or audience in not only our nation but all other nations as well which intend to increase their revenue from OTT platforms in the years to come.

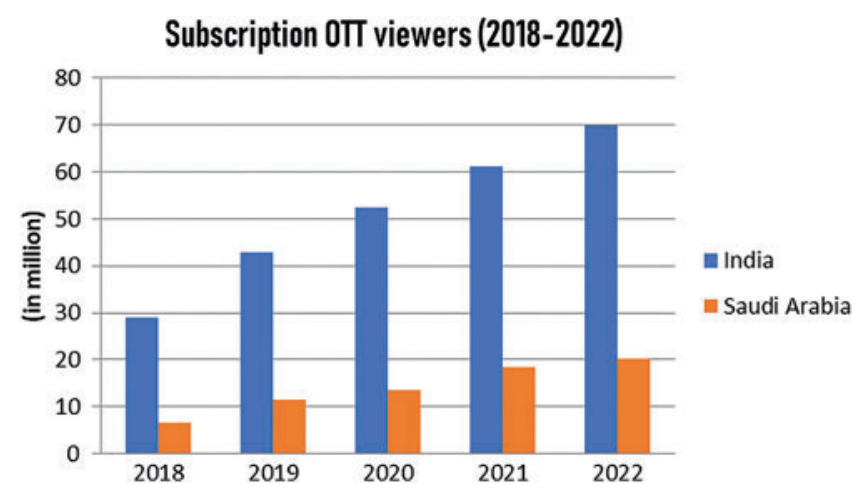

Figure 13: A comparison between the estimated number of subscribed viewers on OTT platforms in India and Saudi Arabia in 2018-2022 


\section{Conclusion and Future Works}

This paper has formulated a solution for honest subscription allocation for OTT platforms using a novel algorithm based on an auction environment. This mechanism is carried by $\mathrm{n}$ bidders who can win incentives for their recommendation on different investment prices. Furthermore, the reward percentage and bidding amount change every $15 \mathrm{~min}$ to establish anonymous voting. The market evaluation metrics and simulations also signify the effectiveness of the model in real-life applications. Hence, designing a similar model suiting cloud computing for budget allocation is our area of immediate concern. Resource provision for different fields might also find usefulness in our proposed research in future implementations.

Funding Statement: The authors received no specific funding for this study.

Conflicts of Interest: The authors declare that they have no conflicts of interest to report regarding the present study.

\section{References}

[1] G. S. Nijhawan and S. Dahiya, "Role of COVID as a catalyst in increasing adoption of OTT's in India: A study of evolving consumer consumption patterns and future business scopes," Journal of Content, vol. 12, pp. 298-311, 2020.

[2] E. Sunderavel and L. Elangovan, "Emergence and future of Over-the-top (OTT) video services in India: An analytical research," International Journal of Buissness Management and Social Research, vol. 8, no. 2, pp. 489-499, 2020.

[3] Y. Kwon, "Research on the relationship between the growth of OTT service market and the change in the structure of the Pay-TV market," in 30th European Conf. of the Int. Telecommunications Society (ITS): "Towards a Connected and Automated Society, Helsinki, Finland, pp. 19-38, 2019.

[4] E. Sundaravel and N. Elangovan, "Emergence and future of Over-the-top (OTT) video services in India: An analytical research," International Journal of Business Management and Social Research, vol. 8, no. 2, pp. 489-499, 2020.

[5] M. K. Patel, R. Khadia and G. Awasya, "A study: OTT viewership in lockdown and viewer's dynamic watching experience," International Journal on Transformations of Media, vol. 5, no. 2, pp. 10-22, 2020.

[6] R. Ota, S. S. Ray and A. Chandra, "An analysis of customer preference towards OTT platform during a pandemic: A special reference to Jamshedpur market," Science Technology and Development Journal, vol. 9, no. 6, pp. 192-202, 2020.

[7] T. Reshma and K. Chaithra, "Proliferation of OTT apps in India: An empirical study of OTT apps and its impact on college studets," International Journal of Research and Analytical Review, vol. 7, no. 1, pp. 427-434, 2020.

[8] T. Iwata, K. Saito and T. Yamada, "Recommendation method for extending subscription period," in 12th ACM SIGKDD Int. Conf. on Knowledge Discovery and Data Mining, KDD 2006, New York, NY, USA, pp. 574-579, 2006.

[9] P. Belleflamme and M. Peitz, "Inside the engine room of digital platforms: Reviews, ratings, and recommendations," Social Science Research Network Electronic Journal, vol. 42, no. 2, pp. 267, 2018.

[10] L. Sharma and A. Gera, "A survey of recommendation system: Research challenges," International Journal of Engineering Trends and Technology, vol. 4, no. 5, pp. 1989-1992, 2013.

[11] J. S. Breese, D. Heckerman and C. Kadie, "Empirical analysis of predictive algorithms for collaborative filtering," in Fourteenth Conf. on Uncertainty in Artificial Intelligence (UAI'98), Madison, Wiscosin, USA, University of Wiscosin Business School, pp. 43-52, 1998.

[12] A. Jain, V. Jain and N. Kapoor, "A literature survey on recommendation system based on sentimental analysis, Advanced Computational Intelligence," An International Journal (ACII), vol. 3, no. 1, pp. 25$36,2016$. 
[13] M. Chen, A. Beutel, P. Covington, S. Jain, F. Belletti et al., "Top-K Off-Policy correction for a REINFORCE Recommender System," in WSDM'19: Twelfth ACM Int. Conf. on Web Search and Data Mining, Melbourne, Australia, pp. 456-464, 2019.

[14] N. Nisan, T. Roughgarden, E. Tardos and V. V. Vazirani, "Combinatorial Auctions," in Algorithmic Game Theory, $1^{\text {st }}$ ed., vol. 1. New York, USA: Cambridge University Press, pp. 267-298, 2007.

[15] R. P. McAfee and J. McMillan, "Auctions and Bidding," Journal of Economic Literature, vol. 25, no. 2, pp. 699-738, 1987.

[16] P. Milgrom, "Putting auction theory to work: The simultaneous ascending auctions," Journal of Political Economy, vol. 108, no. 2, pp. 245-272, 2000.

[17] S. D. Jap, "The impact of online reverse auction design on buyer-supplier relationships," Journal of Marketing, vol. 71, no. 1, pp. 146-159, 2007.

[18] F. Gul and E. Stacchetti, "The english auction with differentiated commodities," Journal of Economic Theory, vol. 92, no. 1, pp. 66-95, 2000.

[19] D. Nakajima, "First-price auctions, Dutch auctions, and buy-it-now prices with Allais paradox bidders: Auctions and prices with allais bidders," Theoretical Economics, vol. 6, no. 3, pp. 473-498, 2011.

[20] P. Emerson, "The original Borda count and partial voting," Social Choice and Welfare, vol. 40, no. 2, pp. 353-358, 2013.

[21] T. W. Sandholm, "Distributed rational decision making," in Multiagent Systems: A Modern Approach to Distributed Artificial Intelligence. Cambridge, USA: MIT Press, pp. 201-258, 1999.

[22] R. K. Dash, D. C. Parkes and N. R. Jennings, "Computational mechanism design: A call to arms," IEEE Intelligent Systems, vol. 18, no. 6, pp. 40-47, 2003.

[23] J. Kim, S. Kim and C. Nam, "Competitive dynamics in the Korean video platform market: Traditional pay TV platforms vs. OTT platforms," Telematics and Informatics, vol. 33, no. 2, pp. 711-721, 2016. 